\title{
Measurement of the Skin-Liver Capsule Distance on Ultrasound RF Data for 1D Transient Elastography
}

\author{
Stéphane Audière ${ }^{1,2}$, Maurice Charbit ${ }^{1}$, Elsa D. Angelini ${ }^{1}$, \\ Jennifer Oudry ${ }^{2}$, and Laurent Sandrin ${ }^{2}$ \\ ${ }^{1}$ Institut Telecom, Telecom ParisTech, CNRS LTCI, Paris, France \\ ${ }^{2}$ Echosens, Research and Development Department, Paris, France
}

\begin{abstract}
Vibration-controlled transient elastography (VCTE ${ }^{\mathrm{TM}}$ ) technique is routinely used in clinical practice to assess non-invasively the liver stiffness which is correlated to hepatic fibrosis. Adequate use of the VCTE ${ }^{\mathrm{TM}}$ probe requires the knowledge of the distance between the skin and the liver parenchyma. This paper compares two methods to estimate this distance using spatial variations of the spectral content of ultrasound radiofrequency (RF) lines, obtained from a probe consisting of a single element ultrasound transducer placed in front of the liver right lobe. Results on a database of 188 patients, including normal-weight and obese persons, show that the spectral variance can accurately discriminate the subcutaneous fat from the liver tissue. The proposed algorithm works in real-time and is suitable for VCTETM scanning protocol setup.
\end{abstract}

Keywords: ultrasound, RF lines, liver, spectral analysis, elastography.

\section{Introduction}

Vibration-controlled transient elastography (VCTETM) technique [1] is routinely used in clinical practice to quantify liver stiffness by measuring the velocity of a lowfrequency shear wave generated and travelling through the liver. It has been demonstrated that stiffness is highly correlated with fibrosis stage assessed by liver biopsy [2]. The VCTETM device can be operated with different 1D-ultrasound probes, operating at different frequencies, depending on the patient morphology. The highest frequency probe provides higher spatial resolution but cannot be used on patients with a thick fat layer, due to frequency-dependent ultrasound attenuation in the fat. Therefore, the choice of the probe during an examination depends on the distance between the probe (in contact with the skin surface) and the liver. This distance corresponds to the subcutaneous thickness, called skin-liver capsule distance (SCD). The SCD is currently measured manually on ultrasound B-mode imaging. Automatic measurement of the SCD is therefore of great interest for VCTE ${ }^{\mathrm{TM}}$ scanning protocol setup to alleviate manual measurements, reduce operator dependency, and standardize the measurement technique. Ultrasound segmentation methods have mainly focused on B-mode imaging given the difficulty in getting access to raw RF data on commercial systems and the complexity of the RF signal content. Unfortunately, B-mode images are formed from the detection of the RF ultrasound line envelopes, which removes 
rich spectral information that could be used to characterize soft tissue properties. Among the few works dedicated to RF-based ultrasound segmentation, we can cite Hammoude et al. [3] who used changes in central frequency due to tissue attenuation to segment the heart muscle but had to face erratic changes in ultrasound signal. Boukerroui et al. [4] proposed a segmentation framework based on gray scale levels and texture analysis from the ultrasound envelope signal, combined with tissue characterization from the analysis of the central frequency spectrum of the ultrasound RF lines. More recently, Dydenko et al. [5] combined RF autoregressive spectrum parameters with the estimation of ultrasound propagation speed to characterize cardiac tissues and obtained good results using the estimated speed variance on in vivo signals. Moradi et al. [6] used the fractal dimension of RF time series for tissue characterisation, but needed high pulse repetition frequency rates. Davignon et al. [7] used the "integrated backScatter coefficient" (IBS) and the average central frequency to improve a segmentation method based on the ultrasound envelope signal. These studies all confirmed that the spectral information of the ultrasound RF lines was adapted for segmentation tasks, providing discriminant information on the acoustic properties of the different tissues.

To our knowledge, few studies have been performed on the automatic measurement of the thickness of the subcutaneous layer, or on the skin-liver capsule distance. $\mathrm{Ng}$ et al. [8] recently showed that changes in the spectral content of $2 \mathrm{D}$-spatial $\mathrm{RF}$ images enabled to accurately measure subcutaneous layer thickness on humans, but required spatial compounding. In this paper, a real-time implementation of the spectral analysis proposed by $\mathrm{Ng}$ et al. [8], but applied to 1D ultrasound RF lines, was evaluated and compared to the exploitation of the IBS parameter from [7].

\section{Materials and Methods}

The SCD includes the layers of tissues connecting the epidermis of the skin surface to the liver. Fibrous membranes of connective tissues may vary in thickness and density between individuals. Furthermore, the fibrous membranes may contain varying proportion of fat and are more echogenic than the liver parenchyma, as illustrated in Fig. 1. Overall the subcutaneous layer has a rather heterogeneous appearance while the liver is rather homogeneous.

\subsection{Spectral RF Analysis}

In most cases, the liver capsule is not precisely detectable on $1 \mathrm{D}$ ultrasound RF lines. Indeed, the direction of the ultrasound beam and the surface of the capsule must be perfectly perpendicular to accurately detect the echo generated by the capsule, as illustrated in Fig. 1. Since it is not possible to visually control the orientation of the VCTE $^{\mathrm{TM}}$ ultrasound beam with respect to the liver surface, the echo amplitude cannot be directly used to determine the SCD. On the other hand, distinction between the subcutaneous layer and the liver can be performed via spectral analysis of the RF lines, based on the fact that ultrasound attenuation is tissue- and frequency-dependent, as detailed below. 


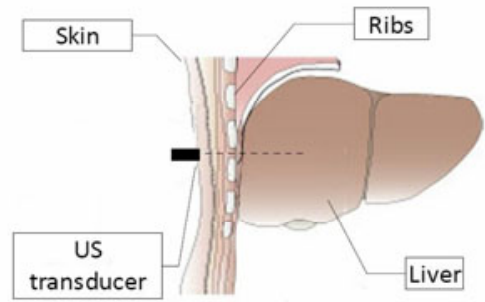

a)

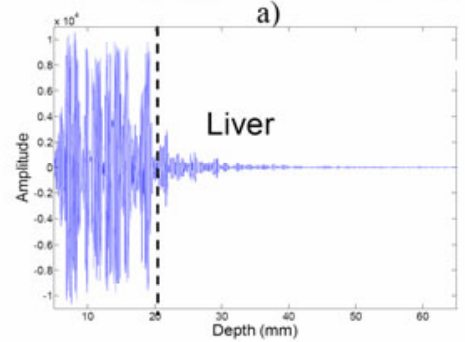

c)

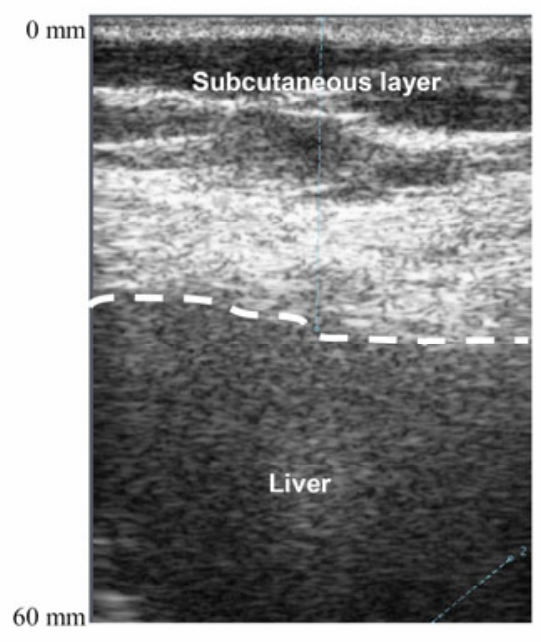

b)

Fig. 1. (a) Positioning of the ultrasound (US) transducer for a VCTETM and B-mode scanning, at the level of the $7^{\text {th }}$ right intercostal space. (b) B-mode image (the position of the capsule is identified with a dashed line.). (c) Ultrasound RF line acquired with the VCTE ${ }^{\mathrm{TM}}$ probe.

The spectrogram $S(f, z)$ of the RF signal at frequency $f$ and at a distance $z$ from the probe is generally modelled as:

$$
S(f, z)=|H(f)|^{2}|R(f)|^{2}|A(f, z)|^{2},
$$

where $H$ is the transfer function of the ultrasonic source, $R$ is the backscatter component from the echoes and $A$ is the attenuation term. In human soft tissues, ultrasound $\mathrm{RF}$ lines are exponentially attenuated in depth, leading to the following model for $A$ :

$$
A(f, z)=e^{-\alpha(f) z},
$$

Within the limited bandwidth of the ultrasound transducer, experimental measures have shown that soft tissues create attenuation phenomena of the RF lines which are linearly proportional to its frequency. Huisman et al. [9] have proposed the following attenuation law:

$$
\alpha(f)=\alpha_{0}+\beta\left(f-f_{c}\right),
$$

where $\alpha_{0}$ is the tissue-specific attenuation coefficient at the central frequency $f_{c}$ of the transducer and $\beta$ is the slope of the frequency-dependent linear attenuation. This attenuation model, exponential in $z$ and with an attenuation coefficient linear in $f$ naturally motivates the choice of a spectral analysis of the ultrasound RF signal for tissue characterization (different tissues being encountered along the $z$-axis).

RF Spectral parameters: Two parameters were calculated from the spectrograms of acquired RF lines: the total energy reflected by the tissues (measured with the IBS) and the spectral variance. The IBS was proposed in [7] as a suitable discriminant 
parameter for tissue characterization. In our case, the tissues constituting the subcutaneous layer are hyper-echogenic relative to the liver parenchyma, leading to potentially higher IBS values. The spectral variance $\sigma^{2}$ characterizes the local variation of the centroid frequency content of the spectrogram. Both can be calculated with the moment method, as proposed in [10], IBS corresponding to $m_{0}$ and the spectral variance being computed as:

$$
\sigma^{2}(z)=\frac{m_{2}(z)}{m_{0}(z)}-\left(\frac{m_{1}(z)}{m_{0}(z)}\right)^{2},
$$

where

$$
m_{n}(z)=\int_{B W} f^{n} S(f, z) d f,
$$

with $B W$ being the useful bandwidth of the transducer. The spectral variance $\sigma^{2}$ does not depend on the echogeneity difference between tissues (i. e. RF signal strength), but rather on the heterogeneity of the tissues. From Eq. (3), $\sigma^{2}$ is also independent of $\alpha_{0}$ and $f_{c}$ but only depends on the tissue-dependent attenuation coefficient $\beta$.

\subsection{Ultrasound RF Scanning Setup and Procedure}

The VCTETM device used in this study was composed of a probe containing a lowfrequency vibrator, an ultrasonic transducer operating, depending on the probe, at 2.5 $\mathrm{MHz}$ or $3.5 \mathrm{MHz}$, a dedicated electronic system and a control unit. The sampling frequency of the ultrasound signal was $50 \mathrm{MHz}$ with a 14-bit resolution. A single ultrasound element was used both as an emitter and a receiver. For each volunteer, 400 ultrasound RF lines were acquired, along a fixed scan line, at a pulse repetition frequency of $20 \mathrm{~Hz}$ during $20 \mathrm{~s}$.

In this study, the following acquisition protocol was used: the liver was first identified with B-mode ultrasound images and the skin capsule distance was manually measured on the images (as illustrated in Fig. 1). The VCTE ${ }^{\mathrm{TM}}$ probe was then placed at the same scanning location, but with the application of a controlled pressure on the skin surface of 20 to $30 \mathrm{kPa}$, for RF recording in elastography scanning mode. Application of such pressure limits probe motion while the patient breathes.

A database was acquired with the two VCTETM probes on 188 volunteers: $85 \%$ with normal weights and $15 \%$ of obese (corresponding to a body mass index (BMI) $>30 \mathrm{~kg} / \mathrm{m}^{2}$ ). The ultrasound RF lines were acquired for a depth range between 5 and $65 \mathrm{~mm}$, to accommodate for the variability of the skin-liver capsule distance within the volunteers population.

\section{Results and Discussion}

\subsection{Spectral Analysis Setup}

For each RF line, the spectrogram was computed with short-time Fourier transform (STFT). Spatial windows of length $12 \lambda$ were used for the STFT computation, where $\lambda$ represents the wavelength (and $2 \lambda$ the pulse length) of the emitted ultrasound signal 


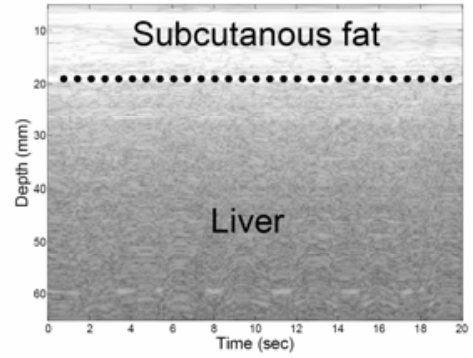

a)

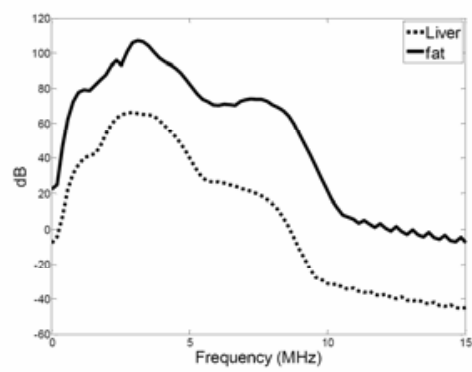

b)

Fig. 2. (a) Series of RF lines acquired with a VCTETM probe on a moderately obese volunteer. (b) Averaged localized spectrogram profiles inside the liver and inside the subcutaneous layer.

(estimated for an average ultrasound velocity $c$ set to $1540 \mathrm{~m} / \mathrm{s}$ for human soft tissues). This spatial window size, which sets the axial precision of the spectrogram, was chosen to optimize the tradeoff between frequency and temporal resolution. Overlaps of $75 \%$ and a weighting Blackman window were applied. With these parameters, we considered that the spectrogram computation had a precision of $3 \lambda$ (i.e. 3 times the axial resolution of the US transducer).

In Fig. 2, an example of a series of temporal ultrasound RF lines acquired with a fixed-position VCTETM $3.5 \mathrm{MHz}$ probe is provided. The volunteer was a male patient measuring $1.67 \mathrm{~m}$ and weighing $82 \mathrm{~kg}\left(\mathrm{BMI}=30 \mathrm{~kg} / \mathrm{m}^{2}\right.$, and B-mode $\mathrm{SCD}=20 \mathrm{~mm}$, corresponding to moderate obesity). Averaged profiles (over $0.5 \mathrm{~s}$ ) of localized spectrogram data for the subcutaneous layer $(z=10 \mathrm{~mm})$ and inside the liver $(z=40 \mathrm{~mm})$ are shown on Fig. 2b. Spectral analysis of the spectrograms, to extract the IBS and spectral variance, was performed on a series of consecutive 400 RF lines acquired over 20 $\mathrm{s}$, maintaining the probe in a fixed position. Spatio-temporal maps of these parameters were computed and a binarization of these maps was applied, based on Otsu's thresholding, to separate the subcutaneous layer from the liver tissue. The series of SCD measures was then combined to provide a robust estimate and compensate for potential tissue interface motion during breathing and to attenuate the effects of noisy components. Averaging of the SCD measures over the series of RF lines was performed in two steps: (1) local averaging over temporal windows of $2 \mathrm{~s}$ (approximate breathing period), (2) hysteresis thresholding for outliers removal on the temporal profile of the SCD values and selection of the SCD median value. For real-time computation during VCTE ${ }^{\mathrm{TM}}$ scanning, these computations are performed in temporal streaming mode, with SCD measures refreshed every $0.5 \mathrm{~s}$.

Parametric maps of the normalized IBS and the normalized spectral variance parameters are illustrated in Fig. 3, along with the corresponding Otsu's thresholding results, for two volunteers. We can clearly observe on the normal-weight volunteer the effect of the breathing on the localisation of the capsule interface, while we don't observe such effect on the obese volunteer. On the normal-weight volunteer, with higher temporal variability on SCD values, the average measures were the following: $\mathrm{SCD}_{\mathrm{IBS}}=12.08 \pm 0.62 \mathrm{~mm}, \mathrm{SCD}_{\sigma^{2}}=13.76 \pm 0.52 \mathrm{~mm}$, while the B-mode measure was $\mathrm{SCD}_{\mathrm{B} \text {-mode }}=13 \mathrm{~mm}$. Maximum and minimum $\mathrm{SCD}$ values over the temporal series 


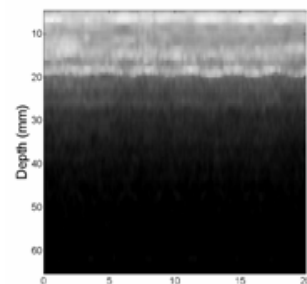

Time (sec)

a)

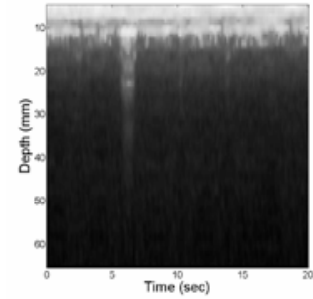

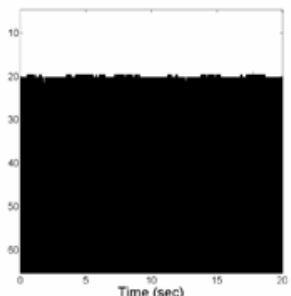

b)

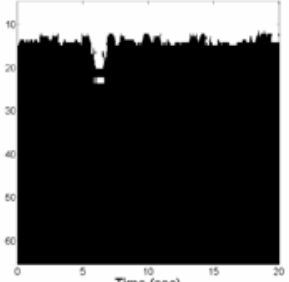

Time (sec)

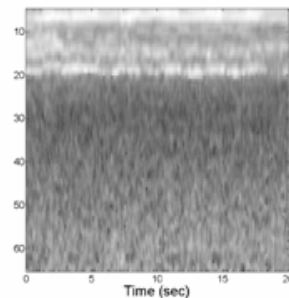

c)

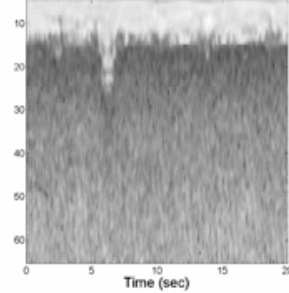

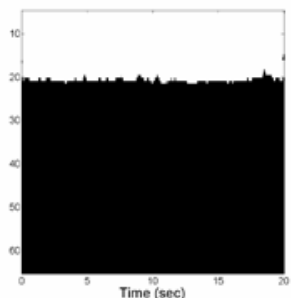

d)

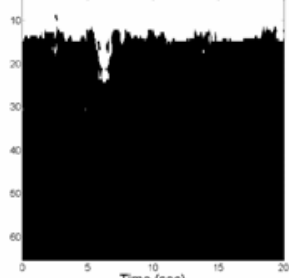

Time (sec)

Fig. 3. Spectral analysis on an obese volunteer (top row) and a normal weight volunteer (bottom row). Normalized parametric maps of the IBS (a) the spectral variance (c) are plotted along with the corresponding thresholding results $(b-d)$.

were: $\mathrm{SCD}_{\mathrm{IBS}}=[11.07 \mathrm{~mm} 13.09 \mathrm{~mm}]$ and $\mathrm{SCD}_{\sigma^{2}}=[12.41 \mathrm{~mm}, 14.43 \mathrm{~mm}]$. This corresponds to lateral liver displacement, relative to the skin surface, of $2 \mathrm{~mm}$ which correlates well with MRI-based findings reported in [11].

\subsection{Quantitative Evaluation of the SCD Measures}

SCD measures based on both spectral parameters were first evaluated on an in vitro experiment to measure the layer thickness on subcutaneous pig tissues, as illustrated in Fig. 4. A pig meat sample made of fat and muscle was placed on top of a gelatin cylinder phantom mimicking the liver acoustic properties.

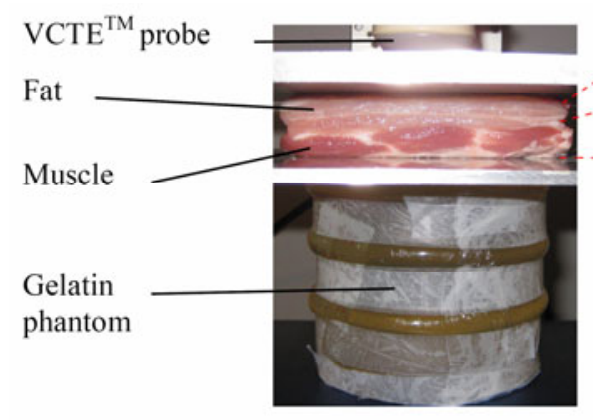

a)

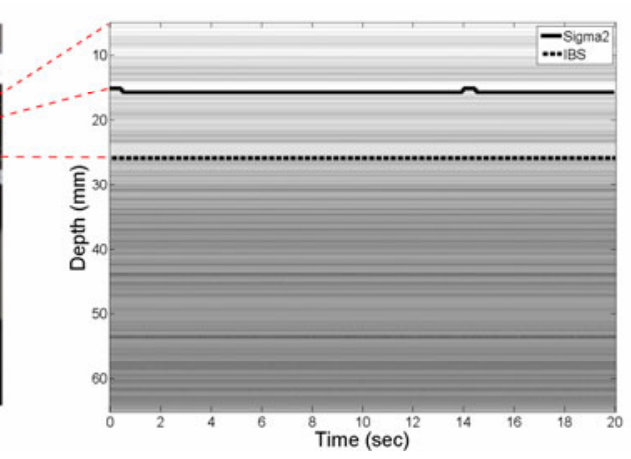

b)

Fig. 4. In vitro experiment on pig tissues. (a) Experimental scanning setup. (b) Temporal series of RF lines. The black line on the image represents the SCD values calculated with the $\mathrm{s}^{2} \mathrm{pa}-$ rameter (solid) and the IBS parameter (dashed). 
The subcutaneous fat layer thickness directly measured on the sample was $13.4 \mathrm{~mm}$. The algorithm provided a temporal average measure of $15 \mathrm{~mm}$ for the $\sigma^{2}$ parameter and $25 \mathrm{~mm}$ for the IBS parameter, both estimations having a spatial precision of 1.25 $\mathrm{mm}$ (for the algorithm). This experiment, performed on high-quality RF lines, showed that the IBS parameter completely failed to provide an accurate fat layer thickness measure, while the $\sigma^{2}$ parameter provided an accurate estimate, within the precision of the method. This experiment highlights the superiority of the spectral variance parameter to distinguish fat from muscle and to be less sensitive to depth-attenuation which is the predominant advantage on in vivo RF data.

\subsection{Qualitative Comparison of SCD Measures on Volunteers}

SCD values measured with the three probes (1 B-mode and $\left.2 \mathrm{VCTE}^{\mathrm{TM}}\right)$, and averaged over the whole population or separately over the normal-weight $\left(\mathrm{SCD}_{\mathrm{B} \text {-mode }}<20 \mathrm{~mm}\right)$ and the over-weight population $\left(\mathrm{SCD}_{\mathrm{B}-\text { mode }}>20 \mathrm{~mm}\right)$, are reported in Table 1 . For the spectral estimations, the algorithm precision of $3 \lambda$ corresponded to $1.8 \mathrm{~mm}$ for the 2.5 $\mathrm{MHz}$ probe and $1.3 \mathrm{~mm}$ for the $3.5 \mathrm{MHz}$ probe. As expected, the SCD measures were smaller with $\mathrm{VCTE}^{\mathrm{TM}}$ probes which apply more pressure on the skin. The range of differences confirmed that $\mathrm{SCD}_{\mathrm{B}-\text { mode }}$ cannot be directly mapped to setup the scanning protocol with the $\mathrm{VCTE}^{\mathrm{TM}}$ probes. The two $\mathrm{VCTE}^{\mathrm{TM}}$ probes should provide similar SCD values, which was only the case with the $\sigma^{2}$ parameter. The IBS parameter estimated systematically lower SCD values with up to $3.7 \mathrm{~mm}(30 \%)$ of measure discrepancies between probes, for the over-weight population.

Focusing on the normal-weight population (SCD $<20 \mathrm{~mm}$ ), the differences between the B-mode and the VCTETM-based measures should be small, given the little deformability of the subcutaneous tissues. Regarding this point, we observed that the similarity of measures was better for the $\sigma^{2}$ parameter than for the IBS. We also observed that the IBS parameter systematically underestimated the subcutaneous layer thickness, especially for the $3.5 \mathrm{MHz}$ probe. Finally, we observed that higher correlations (c.f. p-values) were obtained for the $\sigma^{2}$ parameter, especially for over-weight volunteers. These results overall suggested that this parameter, even though more costly to compute, should be preferred to the IBS parameter.

To evaluate the consistency between SCD measures from the B-mode and the VCTE $^{\mathrm{TM}}$ probes, we performed Kendall's correlation tests on the whole database. Results are reported in Table 2, for the following parameters of the test: $\tau$ is the Kendall's rank correlation coefficient, and p-val is the p-value for the correlation test. The p-values confirmed that the SCD measures provided by the spectral methods were well correlated with B-mode ultrasound measures, while direct comparison was biased, especially on over-weight patients.

Table 1. Average SCD values measured with three probes

\begin{tabular}{cccccc}
\hline SCD $(\mathbf{m m})$ & B-mode & \multicolumn{2}{c}{ VCTETM $^{2.5} \mathbf{~ M H z}$} & \multicolumn{2}{c}{ VCTETM 3.5 MHz } \\
\hline & & $\boldsymbol{\sigma}^{\mathbf{2}}$ & IBS & $\boldsymbol{\sigma}^{\mathbf{2}}$ & IBS \\
All & $17.2 \pm 5.9$ & $16.0 \pm 4.3$ & $12.2 \pm 3.9$ & $15.6 \pm 4.3$ & $10.7 \pm 2.6$ \\
$<20$ & $14.4 \pm 3.7$ & $14.7 \pm 3.2$ & $11.1 \pm 2.8$ & $14.5 \pm 3.7$ & $10.2 \pm 2.3$ \\
$>20$ & $25.1 \pm 6.1$ & $20.4 \pm 4.6$ & $15.9 \pm 4.8$ & $19.4 \pm 4.1$ & $12.2 \pm 3.0$ \\
\hline
\end{tabular}


Table 2. Kendall's correlation test between B-mode and VCTETM SCD measures

\begin{tabular}{ccclllll}
\hline Probe & & \multicolumn{2}{c}{ All SCD } & \multicolumn{2}{c}{ SCD $_{\text {B-mode }} \leq 20 \mathrm{~mm}$} & SCD $_{\text {B-mode }}>20 \mathrm{~mm}$ \\
\hline 2.5 & $\sigma^{2}$ & $\tau=0.6$ & p-val $=2.10^{-24}$ & $\tau=0.5$ & p-val $=6.10^{-15}$ & $\tau=0.4$ & p-val $=2.10^{-3}$ \\
$\mathrm{MHz}$ & IBS & $\tau=0.4$ & p-val $=6.10^{-16}$ & $\tau=0.3$ & p-val $=1.10^{-7}$ & $\tau=0.3$ & p-val $=9.10^{-3}$ \\
\hline 3.5 & $\sigma^{2}$ & $\tau=0.5$ & p-val $=3.10^{-18}$ & $\tau=0.4$ & p-val $=6.10^{-12}$ & $\tau=0.2$ & p-val $=5.10^{-3}$ \\
MHz & IBS & $\tau=0.3$ & p-val $=2.10^{-9}$ & $\tau=0.3$ & p-val $=1.10^{-6}$ & $\tau=0.1$ & p-val $=0.19$
\end{tabular}

\section{Conclusion}

This paper presented the evaluation of a spectral analysis approach to segment ultrasound RF lines and measure subcutaneous layer thickness, for liver scanning, on a large database of subjects. A correlation study was performed to compare thickness measures with an imaging B-mode ultrasound probe. The spectral analysis was tailored to the specific scanning and acquisition setup of a $\mathrm{VCTE}^{\mathrm{TM}}$ probe, involving the tuning of the spatial window length, the weighting window and the spectral bandwidth. In vitro experiment on pig tissues reported a precision of measure of 1 to $2 \mathrm{~mm}$.

\section{References}

1. Sandrin, L., Fourquet, B., Hasquenoph, J.-M., Yon, S., Fournier, C., Mal, F., Christidis, C., Ziol, M., Poulet, B., Kazemi, F.: Transient elastography: A new noninvasive method for assessment of hepatic fibrosis. Ultrasound in Medicine and Biology 29(12), 1705-1713 (2003)

2. Castera, L., Vergniol, J., Foucher, J., Bail, B.L., Chanteloup, E., Haaser, M., Darriet, M., Couzigou, P., Ledinghen, V.d.: Prospective comparison of transient elastography, Fibrotest, APRI, and liver biopsy for the assessment of fibrosis in chronic hepatitis C. Gastroenterology 128(2), 343-350 (2005)

3. Hammoude, A.: Edge detection in ultrasound images based on differential tissue attenuation rates. Ultrasonic Imaging 21(1), 31-42 (1999)

4. Boukerroui, D., Basset, O., Baskurt, A., Gimenez, G.: A multiparametric and multiresolution segmentation algorithm of 3-d ultrasonic data. IEEE Transactions on Ultrasononics, Ferroelectrics and Frequency Control 1(48), 64-77 (2001)

5. Dydenko, I., Friboulet, D., Gorce, J.-M., D’hooge, J., Bijnens, B., Magnin, I.E.: Towards ultrasound cardiac image segmentation based on the radiofrequency signal. Medical Image Analysis 7(3), 353-367 (2003)

6. Moradi, M., Mousavi, P., Abolmaesumi, P.: Tissue characterization using fractal dimension of high frequency ultrasound RF time series. In: Ayache, N., Ourselin, S., Maeder, A. (eds.) MICCAI 2007, Part II. LNCS, vol. 4792, pp. 900-908. Springer, Heidelberg (2007)

7. Davignon, F., Deprez, J.F., Basset, O.: A parametric imaging approach for the segmentation of ultrasound data. Ultrasonics 43(10), 789-801 (2005)

8. Ng, J., Rohling, R., Lawrence, P.D.: Automatic measurement of human subcutaneous fat with ultrasound. IEEE Transactions on Ultrasononics, Ferroelectrics and Frequency Control 56(8), 1642-1653 (2009)

9. Huisman, H.J., Thijssen, J.M.: Precision and accuracy of acoustospectrographic parameters. Ultrasound in Medicine \& Biology 22(7), 855-871 (1996)

10. Fink, M., Hotteir, F., Cardoso, J.F.: Ultrasonic signal processing for in vivo attenuation measurement: Short time Fourier analysis. Ultrasonic Imaging 5(2), 117-135 (1983)

11. Rohlfing, T., Maurer Jr., C.R., O’Dell, W.G., Zhong, J.: Modeling liver motion and deformation during the respiratory cycle using intensity-based free-form registration of gated MR images. In: SPIE Medical Imaging, San Diego, CA, USA (2004) 